\section{First Expansion of the Public Tomato Brown Rugose Fruit Virus (ToBRFV) Nextstrain Build; Inclusion of New Genomic and Epidemiological Data}

\author{
Bart T. L. H. van de Vossenberg, ${ }^{1, \dagger}$ Thikra Dawood, ${ }^{1}$ Marek Woźny, ${ }^{2}$ and Marleen Botermans ${ }^{1}$ \\ ${ }^{1}$ National Reference Centre of plant health, Dutch National Plant Protection Organization, \\ Geertjesweg 15, 6706EA Wageningen, The Netherlands \\ ${ }^{2}$ Main Inspectorate of Plant Health and Seed Inspection, Żwirki i Wigury 73, 87-100 Toruń, Poland
}

\begin{abstract}
Tomato brown rugose fruit virus (ToBRFV) is a tobamovirus that was first detected in Israel and Jordan following an outbreak of a new disease infecting tomato in 2014. Since then, the virus has been reported from all continents except Oceania and Antarctica. In response to the first finding of the virus in The Netherlands, the Dutch National Plant Protection Organization created a ToBRFV Nextstrain build (v1). In this report, we announce 47 new (near) complete ToBRFV genomes and the generation of the new ToBRFV Nextstrain (v2) build containing 118 ToBRFV genomes with associated geographic and epidemiological data. Examples of utilization of the genomic sequences are presented, and we report the first sequence from South America and present a novel hypothesis on the possible ToBRFV center of origin.
\end{abstract}

\section{Announcement}

Tomato brown rugose fruit virus (ToBRFV) is a tobamovirus that was first detected in Israel and Jordan following an outbreak of a new disease infecting tomato (Solanum lycopersicum) in 2014 (Luria et al. 2017; Salem et al. 2016). In addition to tomato, the virus has been reported to infect pepper crops (Capsicum spp.) (Cambrón-Crisantos et al. 2019; Panno et al. 2020; Salem et al. 2020) and, under laboratory conditions, several other plant species such as Petunia sp. and S. nigrum (Luria et al. 2017). Since its first description by Salem and colleagues in 2016, the virus has been reported from all continents except Oceania and Antarctica. In the European Union, ToBRFV is currently regulated as part of EU Commission implementing decision 2020/ 1191 (Anonymous 2020). Following the first finding of the virus in The Netherlands, an interactive ToBRFV Nextstrain build was created to identify possible links between ToBRFV genotypes, epidemiological traits, and distribution (van de Vossenberg et al. 2020). The ToBRFV Nextstrain build published by van de Vossenberg et al. (2020) is referred to as version 1 (v1). In this report, we announce the release of 47 new (near) complete ToBRFV genome sequences with associated metadata, and the second version of the Nextstrain ToBRFV build, which can be accessed via https://nextstrain.nrcnvwa.nl/ToBRFV/20201206.

For the generation, assembly, and annotation of the sequences of (near) complete ToBRFV genomes in this announcement, the methodology described by (van de Vossenberg et al. 2020) was followed. In short, RNA was extracted from fruit, leaves, sepals, stems, or seed of S. lycopersicum or Capsicum spp. using the Sbeadex Maxi Plant Kit (LCG Genomics) on the automated KingFisher Flex 96 platform (Thermo Fisher). Samples that produced cycle quantification values lower than 23 (fruit, fruit sepals, and leaves) or 25 (seed) with one of the real-time reverse-transcription PCR reactions based on primers and probes described by ISHI-VEG (ISF 2019) were selected for Illumina sequencing. De novo assembled and annotated ToBRFV

${ }^{\dagger}$ Corresponding author: B. T. L. H. van de Vossenberg; b.t.I.h.vandevossenberg@nvwa.nl

The author(s) declare no conflict of interest.

Accepted for publication 20 April 2021.

(c) (1) () $\odot$ Copyright (C) 2021 The Author(s). This is an open access article distributed under the CC BY-NC-ND 4.0 International license.

\section{Keywords}

data sharing, epidemiology, genomics, track and trace, virology 
sequences obtained from at least $2 \mathrm{~Gb}$ of 150-bp paired-end plant ribosomal RNA depleted RNA sequencing data were submitted in NCBI GenBank under accessions MW314091 to MW314137. The corresponding sequence read archives were deposited in the European Nucleotide Archive (ENA) under accessions ERR5032738 to ERR5032791. An overview of data associated with the newly generated sequences is shown in Table 1.

Genomic ToBRFV sequences from this report; eight public NCBI GenBank sequences of (near) complete ToBRFV genomes released between November 2019 and October 2020MN013187, MN013188, MN549394, MN549395, MN549396, MN815773 (Beris et al. 2020), MT002973 (Chanda et al. 2020), and MT107885 (Fidan 2020)—and the sequences and metadata from the first version of the ToBRFV Nextstrain build (van de Vossenberg et al. 2020) were

Table 1. Overview of data associated with (near) complete Tomato brown rugose fruit virus (ToBRFV) genome sequences generated in this announcement $^{\mathrm{a}}$

\begin{tabular}{|c|c|c|c|c|c|c|c|c|c|c|}
\hline \multirow[b]{2}{*}{ Sample } & \multirow[b]{2}{*}{ Collection date } & \multirow[b]{2}{*}{ Country } & \multirow[b]{2}{*}{ Host } & \multirow{2}{*}{$\begin{array}{l}\text { Material } \\
\text { sampled }\end{array}$} & \multicolumn{2}{|c|}{ Real-time RT-PCR results } & \multirow{2}{*}{$\begin{array}{l}\text { Number } \\
\text { of reads }\end{array}$} & \multirow[b]{2}{*}{$\operatorname{ARC}(x)^{b}$} & \multirow[b]{2}{*}{$\begin{array}{c}\text { SRA } \\
\text { accession }\end{array}$} & \multirow{2}{*}{$\begin{array}{c}\text { NCBI } \\
\text { accession }\end{array}$} \\
\hline & & & & & CSP1325 & CaTa28 & & & & \\
\hline 32607982 & 18 Feb 2019 & Egypt & Solanum lycopersicum & Fruit & NA & NA & $28,820,766$ & 350,900 & ERR5032774 & MW314092 \\
\hline 33314743 & 25 Nov 2019 & Egypt & S. lycopersicum & Fruit & NA & NA & $47,007,964$ & 384,951 & ERR5032756 & MW314094 \\
\hline 36689655 & 20 Dec 2019 & Jordan & S. lycopersicum & Seed & 3.7 & 4.8 & $24,396,862$ & 460,152 & $\begin{array}{l}\text { ERR5032740; } \\
\text { ERR5032746 }\end{array}$ & MW314107 \\
\hline 33314313_B & 18 Nov 2019 & Egypt & S. lycopersicum & Fruit & NA & NA & $42,981,588$ & 821,870 & ERR5032757 & MW314093 \\
\hline 33837271_1 & 28 Feb 2020 & Netherlands & S. lycopersicum & Leaves & 8.3 & 5.8 & $26,539,030$ & 118,996 & ERR5032748 & MW314095 \\
\hline 33837271_2 & 28 Feb 2020 & Netherlands & S. lycopersicum & Leaves & 5.0 & 4.7 & $18,241,966$ & 98,161 & ERR5032749 & MW314096 \\
\hline 33837296_1 & 28 Feb 2020 & Netherlands & S. lycopersicum & Leaves & 9.1 & 5.7 & $19,601,282$ & 180,247 & ERR5032750 & MW314097 \\
\hline 33837296_2 & 28 Feb 2020 & Netherlands & S. Iycopersicum & Leaves & 8.7 & 4.7 & $21,865,848$ & 194,936 & ERR5032751 & MW314098 \\
\hline 34169391_1 & 29 Nov 2019 & Netherlands & S. lycopersicum & Sepals & 16.2 & 16.2 & $28,116,288$ & 821 & ERR5032788 & MW314099 \\
\hline 34169391_2 & 29 Nov 2019 & Netherlands & S. lycopersicum & Sepals & 9.6 & 8.8 & $27,282,382$ & 77,202 & ERR5032789 & MW314100 \\
\hline $34169402 \_1$ & 29 Nov 2019 & Netherlands & S. lycopersicum & Leaves & 11.5 & 11.4 & $25,678,314$ & 94,077 & ERR5032790 & MW314101 \\
\hline 34169402_2 & 29 Nov 2019 & Netherlands & S. lycopersicum & Leaves & 12.2 & 11.6 & $30,418,834$ & 123,593 & ERR5032791 & MW314102 \\
\hline 36638057_3 & 16 Apr 2020 & Netherlands & S. lycopersicum & Sepals & 15.4 & 14.8 & $16,464,630$ & 3,101 & $\begin{array}{l}\text { ERR5032764; } \\
\text { ERR5032781; } \\
\text { ERR5032782 }\end{array}$ & MW314103 \\
\hline 36689436_1 & 20 Dec 2019 & Jordan & Capsicum annuum & Seed & 21.0 & 21.5 & $13,729,434$ & 189 & ERR5032755 & MW314104 \\
\hline 36689444_1 & 29 Jan 2020 & Jordan & Capsicum annuum & Seed & 11.3 & 12.1 & $39,559,582$ & 2,896 & ERR5032761 & MW314105 \\
\hline 36689516_1 & 9 Mar 2020 & Netherlands & S. lycopersicum & Seed & 16.2 & 16.5 & $20,132,298$ & 636 & ERR5032758 & MW314106 \\
\hline 36689663_1 & 20 Dec 2019 & Jordan & S. lycopersicum & Seed & 4.1 & 4.6 & $14,981,458$ & 300,206 & $\begin{array}{l}\text { ERR5032741; } \\
\text { ERR5032747 }\end{array}$ & MW314108 \\
\hline 36689751_1 & 20 Dec 2019 & Jordan & S. lycopersicum & Seed & 13.2 & 13.2 & $22,898,576$ & 11,189 & ERR5032738 & MW314109 \\
\hline 36689794_1 & 20 Dec 2019 & Jordan & S. Iycopersicum & Seed & 2.1 & 2.1 & $13,455,782$ & 282,125 & ERR5032739 & MW314110 \\
\hline 36783571_2 & 31 Mar 2020 & Peru & S. lycopersicum & Seed & 14.4 & 13.7 & $40,560,220$ & 6,832 & ERR5032765 & MW314111 \\
\hline 36783668_2 & 7 Apr 2020 & Netherlands & S. lycopersicum & Seed & 20.0 & 21.4 & $46,172,096$ & 34 & ERR5032766 & MW314112 \\
\hline 36783756_2 & 4 Jun 2020 & Israel & Capsicum annuum & Seed & 12.1 & 12.5 & $14,073,884$ & 672 & ERR5032767 & MW314113 \\
\hline 36783860_1 & 14 Apr 2020 & China & S. lycopersicum & Seed & 10.0 & 10.1 & $22,321,696$ & 27,518 & ERR5032760 & MW314114 \\
\hline 36810152_2 & 19 Mar 2020 & Israel & S. lycopersicum & Seed & 16.4 & 17.3 & $18,388,928$ & 9,156 & ERR5032762 & MW314115 \\
\hline 36810152_3 & 19 Mar 2020 & Israel & S. lycopersicum & Seed & 16.5 & 17.4 & $14,611,604$ & 54 & ERR5032763 & MW314116 \\
\hline 36810232_3 & 20 Mar 2020 & Netherlands & S. lycopersicum & Seed & 15.9 & 16.2 & $22,310,498$ & 23,828 & ERR5032759 & MW314117 \\
\hline 38589922_1 & 28 Feb 2020 & Netherlands & S. lycopersicum & Leaves & 8.2 & 5.3 & $23,373,016$ & 231,541 & ERR5032752 & MW314118 \\
\hline 38589922_2 & 28 Feb 2020 & Netherlands & S. lycopersicum & Leaves & 16.6 & 17.1 & $25,839,224$ & 1,795 & ERR5032753 & MW314119 \\
\hline 38589922_3 & 28 Feb 2020 & Netherlands & S. lycopersicum & Leaves & 8.8 & 4.9 & $21,471,486$ & 154,263 & ERR5032754 & MW314120 \\
\hline 39072896_1 & 26 Nov 2019 & Netherlands & S. lycopersicum & Leaves & 10.7 & 10.1 & $24,892,422$ & 108,910 & ERR5032744 & MW314121 \\
\hline 39072896_2 & 26 Nov 2019 & Netherlands & S. lycopersicum & Leaves & 13.1 & 12.3 & $13,127,192$ & 26,779 & ERR5032745 & MW314122 \\
\hline 39563433_3 & 18 May 2020 & Netherlands & S. lycopersicum & Leaves & 13.5 & 13.3 & $15,531,416$ & 452,193 & $\begin{array}{l}\text { ERR5032768; } \\
\text { ERR5032783; } \\
\text { ERR5032784 }\end{array}$ & MW314123 \\
\hline 39774038_1 & 28 Nov 2019 & Netherlands & S. lycopersicum & Leaves & 9.1 & 5.2 & $26,196,904$ & 161,215 & ERR5032786 & MW314124 \\
\hline 39774038_2 & 28 Nov 2019 & Netherlands & S. lycopersicum & Leaves & 5.5 & 4.1 & $25,828,516$ & 330,753 & ERR5032787 & MW314125 \\
\hline 39971816_1 & 25 Nov 2019 & Netherlands & S. lycopersicum & Leaves & 15.1 & 16.2 & $26,630,360$ & 1,167 & ERR5032742 & MW314126 \\
\hline 39971816_2 & 25 Nov 2019 & Netherlands & S. lycopersicum & Leaves & 18.5 & 20.1 & $26,860,324$ & 61 & ERR5032743 & MW314127 \\
\hline 39985935_4 & 22 Jun 2020 & Netherlands & S. lycopersicum & Leaves & 9.5 & 7.4 & $89,389,096$ & $1,535,600$ & $\begin{array}{l}\text { ERR5032769; } \\
\text { ERR5032785 }\end{array}$ & MW314128 \\
\hline 40001980_D & 8 Oct 2020 & Netherlands & S. lycopersicum & Leaves & 11.0 & 10.3 & $18,267,336$ & 62,319 & ERR5032780 & MW314129 \\
\hline 40002000_A & 30 Jul 2020 & Netherlands & S. lycopersicum & Leaves & 12.2 & 12.2 & $16,539,462$ & 75,403 & ERR5032773 & MW314130 \\
\hline 40002166_A & 30 Jun 2020 & Netherlands & S. lycopersicum & Sepals & 3.6 & 2.7 & $70,199,120$ & $1,123,963$ & ERR5032770 & MW314131 \\
\hline 40002350_A & 5 Aug 2020 & Netherlands & S. lycopersicum & Sepals & 4.0 & 5.4 & $24,866,542$ & 377,463 & ERR5032772 & MW314132 \\
\hline 40002377_1A & 3 Sep 2020 & Netherlands & S. lycopersicum & Sepals & 5.4 & 4.0 & $19,760,698$ & 243,797 & ERR5032775 & MW314133 \\
\hline 40002377_2B & 3 Sep 2020 & Netherlands & S. Iycopersicum & Sepals & 5.9 & 4.1 & $19,395,176$ & 299,016 & ERR5032776 & MW314134 \\
\hline 40002377_3C & 3 Sep 2020 & Netherlands & S. lycopersicum & Sepals & 3.1 & 2.9 & $26,646,018$ & 451,723 & ERR5032777 & MW314135 \\
\hline 40002385_A & 15 Sep 2020 & Netherlands & S. lycopersicum & Leaves & 4.3 & 4.7 & $21,505,372$ & 450,466 & ERR5032778 & MW314136 \\
\hline 40002393_C & 5 Aug 2020 & Netherlands & S. lycopersicum & $\begin{array}{l}\text { Stems and } \\
\text { leaves }\end{array}$ & 16.9 & 16.8 & $32,230,150$ & 464 & ERR5032771 & MW314137 \\
\hline 6189975_2 & 19 Oct 2020 & China & S. lycopersicum & Seed & 18.0 & 19.8 & $27,721,922$ & 4,034 & ERR5032779 & MW314091 \\
\hline
\end{tabular}

a SRA = Sequence Read Archive. NA = not available; sample was included for Illumina sequencing based on other methods such as enzymelinked immunosorbent assay, indicator plants, or other real-time reverse-transcription PCR (RT-PCR) tests.

${ }^{\mathrm{b}}$ Average read coverage (ARC) of the (near) complete ToBRFV genome. 
used to create the ToBRFV Nextstrain (v2) build. The new build was generated as described by van de Vossenberg et al. (2020), except for the Augur Export command version (i.e., v2 instead of v1), allowing customization of the webpage. The ToBRFV Nextstrain (v2) build contains 118 genomic sequences from 15 countries from materials sampled between November 2014 and October 2020 (Fig. 1A). New in the ToBRFV Nextstrain (v2) build is information on the country of origin of rootstock and scion seed. In addition, major clades have been defined to facilitate the communication of genomic ToBRFV diversity. The augur input data and scripts are available on GitHub via https://github.com/NPPO-NL/nextstrain-ToBRFV.

The new sequence data and the associated metadata as presented in the ToBRFV Nextstrain (v2) build are instrumental for the plant virological research community to better understand the diversity and epidemiology of ToBRFV in global context. For regulatory bodies, such data can be helpful to take risk-based measures and identify or disqualify potential outbreak sources. An example of the latter is to determine the effect of cleaning and disinfection of affected greenhouses during crop rotation. Dutch fruit companies that were found to have ToBRFV at their premises intensively cleaned and disinfected their facilities during crop rotation. Unfortunately, in some cases, ToBRFV was detected again at these locations 3 to 6 months into the new ToBRFV host production cycle. Sequences generated from those new infections could be used to determine whether companies had not managed to eliminate the virus from their facilities or reintroduced the virus from the same source, or if those companies contracted the virus from another source. Examples of both cases are represented in the ToBRFV Nextstrain (v2) build (Fig. 1B and C). In one case, two identical sequences from clade 6 were obtained following sampling in October 2019 (MN882042 and MN882043) at a production
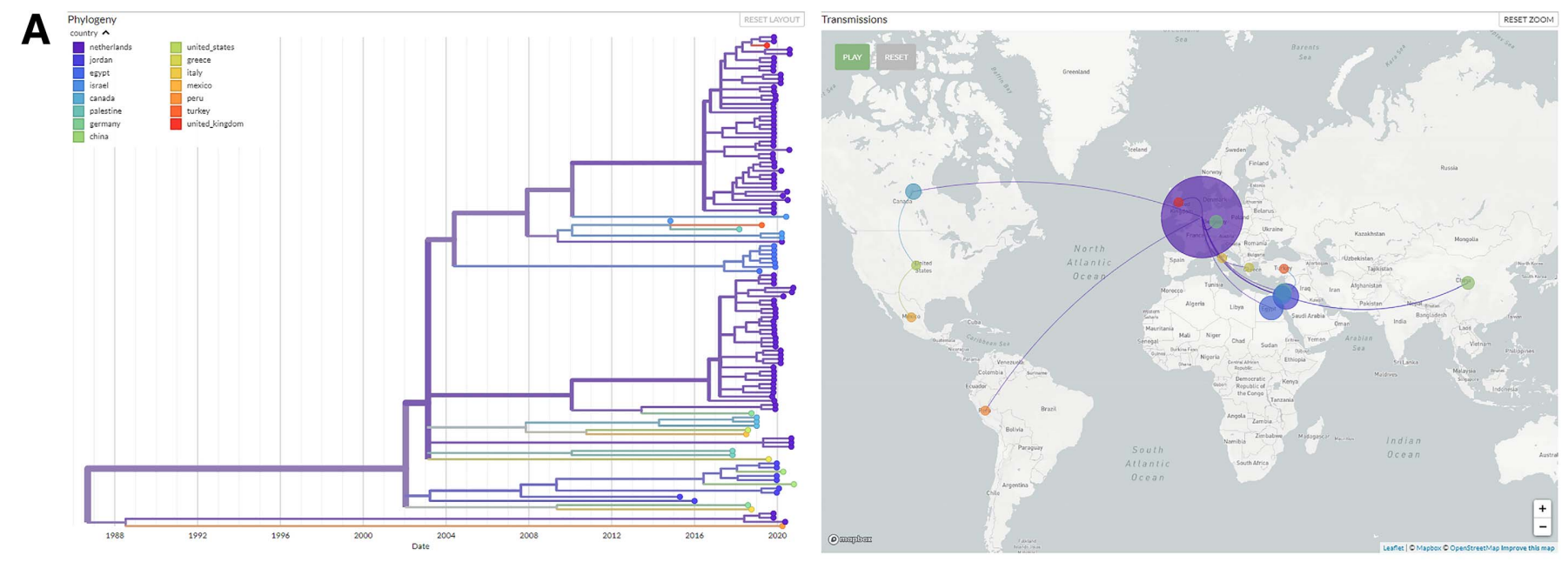

B
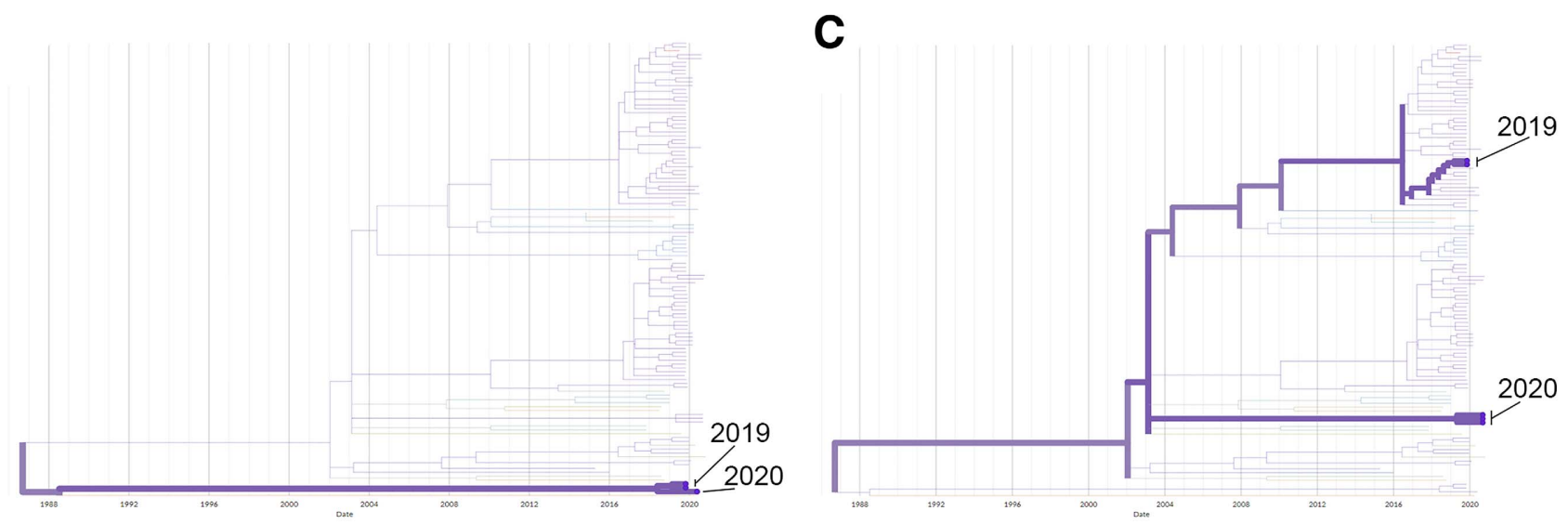

Fig. 1. Tomato brown rugose fruit virus (ToBRFV) Nextstrain v2. A, Genomic epidemiology of 118 (near) complete ToBRFV genomes sampled between October 2014 and October 2020 represented as a time tree (left panel) and the geographic distribution (right panel). Examples of tomato production locations where ToBRFV was found in 2019 and (after intensive cleaning and crop rotation) again in 2020 are presented. B, In one case, a nearly identical sequence was found in the year following the initial infestation and $\mathbf{C}$, in another case, sequences from a different cluster were identified in the year following the initial infestation. 
location in the Dutch municipality of Brielle. After cleaning and crop rotation, ToBRFV was found again in the new tomato crop several months later, producing a sequence nearly identical (MW314123: 3 single-nucleotide polymorphism [SNPs]; 99.95\% sequence similarity) to the previously sequenced material. In this case, it is likely that the company did not manage to fully eliminate the virus from their facilities. In another case, ToBRFV was detected in November 2019 at a production location in the Dutch municipality of Reimerswaal. Sequencing of the obtained material resulted in two identical clade 1 sequences with (MN882059 and MN882060). In September 2020, ToBRFV was detected again at the same production site and, again, three identical sequences were obtained (MW314133, MW314134, and MW314135). However, the 2020 sequences form a unique cluster and differed by 22 SNPs (= 99.65\% sequence similarity) compared with the 2019 sequences. For this production site, it is likely that the virus was introduced anew from another source.

Among the newly generated sequences is one (near) complete ToBRFV genome obtained from tomato seed from Peru (MW314111). This sequence represents the first sequence from a South American country, and is (together with the sequences found at one Dutch outbreak location) early diverging in the RAxML time tree. This early-diverging lineage suggests that more sequence diversity can be expected and, currently, not all intraspecific variation is known. Given the position of the Peruvian sequence in the tree, one could speculate that the center of origin of ToBRFV might not lie in the region from where it was first described (i.e., the Middle East) but could lie in South America. Phylogenetic analyses from tobamoviruses and their solanaceous crops were shown to be congruent (Gibbs et al. 2015), which supports our hypothesis that ToBRFV might originate from the presumed center of origin for the Solanaceae family (i.e., South America) (Olmstead 2013). Because the sequence diversity is expected to be highest at the center of origin, those sequences are expected to be broadly represented in the tree, particularly in the early-diverging branches. With 82 of 118 sequences originating from materials sampled at outbreak locations in The Netherlands, there is a strong sampling bias toward Dutch ToBRFV outbreak locations. To reliably test the South American center-of-origin hypothesis, more sequences from South America and other locations worldwide are needed.

The predictive power of the ToBRFV Nextstrain build will improve with the inclusion of additional genomes and metadata. Therefore, we encourage other organizations to share sequence data or biological materials together with relevant metadata to improve the build and our understanding of the diversity and spread of this important virus.

\section{Data Availability}

The (near) complete ToBRFV genomes assembled and annotated in this study were submitted to NCBI GenBank under accessions MW314091 to MW314137. The corresponding sequence read archives were deposited in ENA under accessions ERR5032738 to ERR5032791. The ToBRFV Nextstrain (v2) build, containing 118 ToBRFV genomes with associated geographic and epidemiological data, is available via https://nextstrain.nrcnvwa.nl/ ToBRFV-internal/20201206, and the underlying Augur input data and scripts are available via the National Plant Protection Organization of The Netherlands (NPPO-NL) GitHub repository via https://github.com/NPPO-NL/nextstrain-ToBRFV.

\section{Acknowledgments}

We thank the NPPO-NL staff members J. Tijssen, M. van Lent, and M. Visser for collecting metadata and providing technical support in updating the NPPO-NL GitHub repository; R. van Velzen (Wageningen University) for providing valuable input on an early version of the draft; and Naktuinbouw for performing the first-line detection tests.

\section{Literature Cited}

Anonymous. 2020. Commission Implementing Regulation (EU) 2020/1191 of August 11, 2020 establishing measures to prevent the introduction into and the spread within the Union of Tomato brown rugose fruit virus (ToBRFV) and repealing Implementing Decision (EU) 2019/1615.

Beris, D., Malandraki, I., Kektsidou, O., Theologidis, I., Vassilakos, N., and Varveri, C. 2020. First report of tomato brown rugose fruit virus infecting tomato in Greece. Plant Dis. 104:2035.
Cambrón-Crisantos, J. M., Rodríguez-Mendoza, J., Valencia-Luna, J. B., AlcasioRangel, S., García-Ávila, C. de J., López-Buenfil, J. A., and Ochoa-Martínez, D. L. 2019. First report of Tomato brown rugose fruit virus (ToBRFV) in Michoacan, Mexico [Primer reporte de Tomato brown rugose fruit virus (ToBRFV) en Michoacán, México]. Rev. Mex. Fitopatol. 37:185-192.

Chanda, B., Rivera, Y., Nunziata, S. O., Galvez, M. E., Gilliard, A., and Ling, K.-S. 2020. Complete genome sequence of a Tomato brown rugose fruit virus isolated in the United States. Microbiol. Resour. Announce. 9:e00630-20. 
Fidan, H. 2020. Tomato brown rugose fruit virus (ToBRFV): Current situation and future prospects. Mediterr. Agric. Sci. 33:43-49.

Gibbs, A. J., Wood, J., Garcia-Arenal, F., Ohshima, K., and Armstrong, J. S. 2015. Tobamoviruses have probably co-diverged with their eudicotyledonous hosts for at least 110 million years. Virus Evol. 1:vev019.

ISF. 2019. Detection of infectious Tomato brown rugose fruit virus (ToBRFV) in tomato and pepper seeds. ISHI-veg, 11. International Seed Federation. https:// www.worldseed.org/wp-content/uploads/2019/09/Tomato-ToBRFV_2019.09.pdf

Luria, N., Smith, E., Reingold, V., Bekelman, I., Lapidot, M., Levin, I., Elad, N., Tam, Y., Sela, N., Abu-Ras, A., Ezra, N., Haberman, A., Yitzhak, L., Lachman, O., and Dombrovsky, A. 2017. A new Israeli Tobamovirus isolate infects tomato plants harboring Tm-22 resistance genes. PLoS One 12:e0170429.
Olmstead, R. G. 2013. Phylogeny and biogeography in Solanaceae, Verbenaceae and Bignoniaceae: A comparison of continental and intercontinental diversification patterns. Bot. J. Linn. Soc. 171:80-102.

Panno, S., Caruso, A. G., Blanco, G., and Davino, S. 2020. First report of Tomato brown rugose fruit virus infecting sweet pepper in Italy. New Dis. Rep. 41:20.

Salem, N., Mansour, A., Ciuffo, M., Falk, B. W., and Turina, M. 2016. A new tobamovirus infecting tomato crops in Jordan. Arch. Virol. 161:503-506.

Salem, N. M., Cao, M. J., Odeh, S., Turina, M., and Tahzima, R. 2020. First report of tobacco mild green mosaic virus and tomato brown rugose fruit virus infecting Capsicum annuum in Jordan. Plant Dis. 104:601.

van de Vossenberg, B. T. L. H., Visser, M., Bruinsma, M., Koenraadt, H. M. S., Westenberg, M., and Botermans, M. 2020. Real-time tracking of Tomato brown rugose fruit virus (ToBRFV) outbreaks in the Netherlands using Nextstrain. PLoS One 15:e0234671. 